\title{
Understanding the invisible hands of sample preparation for cryo-EM
}

\author{
Giulia Weissenberger ${ }^{1,2,3}$, Rene J. M. Henderikx ${ }^{1,2,3}$ and Peter J. Peters $\mathbb{1}^{2} \otimes$
}

\begin{abstract}
Cryo-electron microscopy (cryo-EM) is rapidly becoming an attractive method in the field of structural biology. With the exploding popularity of cryo-EM, sample preparation must evolve to prevent congestion in the workflow. The dire need for improved microscopy samples has led to a diversification of methods. This Review aims to categorize and explain the principles behind various techniques in the preparation of vitrified samples for the electron microscope. Various aspects and challenges in the workflow are discussed, from sample optimization and carriers to deposition and vitrification. Reliable and versatile specimen preparation remains a challenge, and we hope to give guidelines and posit future directions for improvement.
\end{abstract}

$\mathrm{C}$ ryo-EM is providing macromolecular structures with up to atomic resolution at an unprecedented rate. In this technique, electron microscopy images of biomolecules embedded in vitreous, glass-like ice are combined to generate three-dimensional (3D) reconstructions. The detailed structural models obtained from these reconstructions grant insight into the function of macromolecules and their role in biological processes. For example, resolving the structures of tau filaments ${ }^{1}$ and amyloid fibrils ${ }^{2}$ has provided insights into the mechanism of Alzheimer's disease, the most common neurodegenerative disorder. Cryo-EM was also instrumental in resolving the structure of the SARS-CoV-2 spike protein, which helps the virus gain entry to human cells, at a resolution of $3.5 \AA^{3}$. Understanding the process of virus binding to specific receptors on human cells will aid the fabrication of targeted vaccines, drugs and diagnostics ${ }^{4,5}$. Pharmaceutical organizations have already shown a growing interest in the technique to analyze ligand binding, perform conformational studies and carry out drug testing ${ }^{6}$.

The rise in popularity of cryo-EM as a breakthrough structural biology technique comes as a result of a number of technical advancements, often referred to as the 'resolution revolution' (ref. ${ }^{7}$ ). One of the key driving forces has been the introduction of a new generation of cameras with direct electron detection and increased frame rates ${ }^{8}$. The new cameras are able to operate in single-electron counting mode and acquire movies to compensate for movement during imaging. Cryo-electron microscopes have become more amenable to automation through features such as the autoloader, constant-power lenses, mechanical stages with decreased drift and aberration-free image shifting. Software packages allow continuous unsupervised collection of high-quality data over days. Furthermore, data processing has become more robust and user friendly ${ }^{10}$, with automated pipelines being developed to streamline data acquisition and processing ${ }^{11}$. Hardware improvements, primarily aimed at narrowing the energy spread of incident electrons, have brought the achievable resolution to below $1.3 \AA^{12,13}$. While these technical upgrades have made cryo-EM more accessible to structural biologists, sample preparation has emerged as the main impediment in the workflow, leading scientists and engineers to target this hurdle ${ }^{14}$.

Sample preparation in single-particle analysis has two main components: sample optimization and grid preparation. Sample optimization involves specimen purification while maintaining the optimum biochemical state of the sample. Grid preparation describes the steps needed to make a sample suitable for analysis in the microscope. These steps involve chemical or plasma treatment of the grid, sample deposition and vitrification. The first breakthroughs came about from a manual blot-and-plunge method developed in the $1980 \mathrm{~s}^{15}$ that is still being applied to achieve formidable results ${ }^{16-18}$.

As more heterogenous and complex samples are studied, special care is required to maintain their stability ${ }^{19}$. Applying the sample from a bulk solution onto a grid can have an impact on the state of the molecule. When analyzed in the microscope, vitrified samples are often subject to aggregation, preferential orientation or denaturation ${ }^{14}$. These phenomena can be minimized through sample optimization at the biochemical level or through optimization of various parameters in grid preparation. Numerous techniques have been proposed to overcome macromolecular instability; however, the effectiveness of one method over another varies by sample ${ }^{14,20}$. Currently, the results from grid preparation are mostly dependent on user expertise and experience, and this process can become increasingly time consuming and challenging ${ }^{21}$.

The many variables encountered in sample and grid preparation make it difficult to identify cause-and-effect relationships. The researcher only evaluates the sample at the molecular level at the microscope. While the procedure of preparing grids does not require much time, the iterations needed to obtain the right conditions can be time consuming and labor intensive ${ }^{21,22}$. Pragmatically, this makes it difficult to standardize protocols. Although databases have aided in better monitoring of conditions and outcomes ${ }^{23,24}$, quantitative statistics from comparisons between different protocols for grid preparation are still lacking. A structured approach is essential to investigate trends and understand the underlying mechanisms of sample behavior. If, among others, these problems are solved, cryo-EM could become a more versatile and even more dominant technology in structural biology ${ }^{25}$. New research questions will potentially be addressed, in turn aiding the development of novel methodologies as the field grows.

Many steps of the workflow can be manipulated, starting with the sample in solution, the EM grid and the way the sample is deposited and vitrified. In this Review, we explicate the fundamentals of the different stages of sample preparation (Fig. 1). On the basis of these requisites of sample preparation, we delineate the benefits and drawbacks of the available methodologies. 


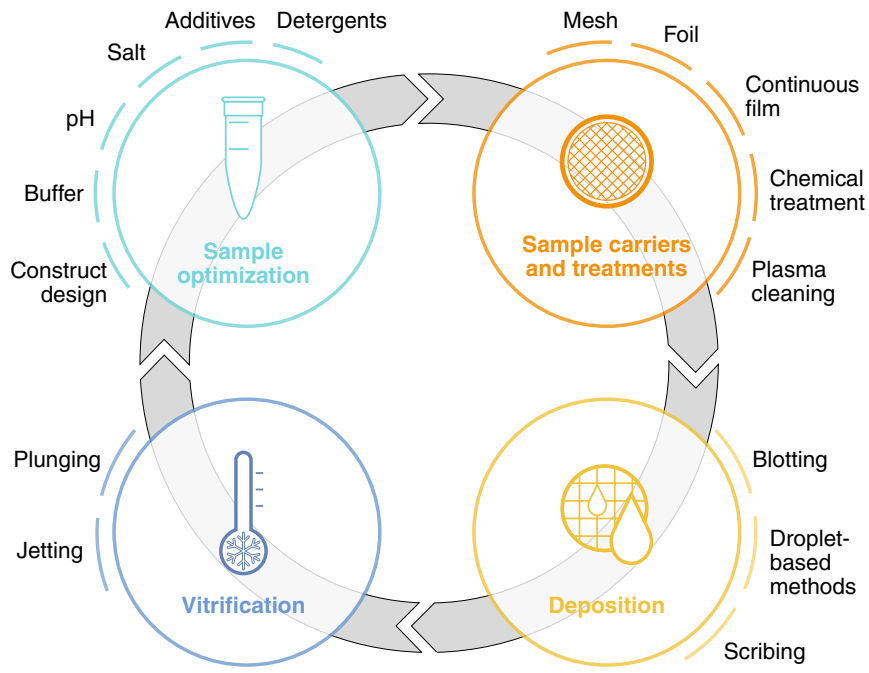

Fig. 1 General overview of the stages involved in sample preparation for cryo-EM. These stages include sample optimization, sample carriers and treatments, deposition and vitrification. Each of these stages is subdivided into options users are faced with in the workflow.

\section{Sample optimization}

An ideal session at the microscope requires a dataset with a good concentration of intact particles embedded as a monolayer in thin ice; the particles are randomly oriented and evenly distributed, and the vitrified specimen is free of contamination. The quest begins in the wet lab where the candidate molecule is identified and the construct is designed. Numerous biochemical assays are performed to isolate and purify the macromolecule from its cellular environment while attempting to emulate its native conditions.

The molecule's native environment is mimicked in bulk solution by tuning the $\mathrm{pH}$ and adding salt, glycerol or other additives. In creating a film of sample solution intended for the microscope, the high surface-to-volume ratio can give rise to challenges in stability. Stability issues can range from preferential orientation to denaturation or aggregation of sample ${ }^{14}$. Samples are often divided into different classes, which include cytosolic and membrane proteins and larger macromolecular protein complexes. Cytosolic, or soluble, proteins have a tendency to remain stable in thin films owing to the hydrophilic amino acids on their external structures. Membrane proteins, on the other hand, often need further stabilization. The exposed hydrophobic amino acids that are naturally anchored within the lipid bilayer make them especially susceptible to the air-water interface. Membrane proteins are stabilized by mimicking the lipid bilayer using detergents, amphipols, nanodiscs and styrene-maleic acid copolymers ${ }^{26}$. These additives form a protective ring around the hydrophobic regions of the sample, with amphipols having a higher affinity than detergents. Lipid nanodiscs and styrene-maleic acid copolymers are more recent developments that have a closer resemblance to the lipid bilayer ${ }^{26}$. By using nanodiscs, different conformations of receptors in pentameric ligand-gated ion channels were resolved, shedding light on the gating mechanism ${ }^{27}$. Another approach to handle fragile proteins is to block the air-water interface. While detergents are often used during the purification process, others are added before deposition of the sample on a grid, shielding it from the interface. Addition of the fluorinated detergent CHAPSO has shown promising effects on particle orientation in the case of bacterial RNA polymerase ${ }^{28}$. To preserve larger structures, chemical cross-linking can be applied to maintain structural integrity. Cross-linking can be done either in solution or using a sucrose gradient ${ }^{29,30}$. However, caution should be taken in rigidifying structures, as flexible domains can be locked in one conformation.
Before introducing a sample to the electron microscope, the sample in solution can be visually assessed by negative staining. This is used for rapid screening before cryo-EM and helps to evaluate the homogeneity of the sample along with its buffer components ${ }^{31}$. While negative staining provides general insight about the molecule, high-resolution details cannot be observed. Despite the benefits of screening, dissociation at the air-water interface ${ }^{32}$ or concentration-dependent aggregation ${ }^{33}$ of molecules can still arise when preparing the same specimen in a vitrified state ${ }^{33}$.

\section{Sample carriers and treatments}

Sample carriers are historically circular EM grids of $3 \mathrm{~mm}$ in diameter that consist of at least two components: a mesh base and foil. The mesh base is made of metal to attain mechanical stability, conduction of the electron beam and heat dissipation ${ }^{34}$. Commercially available meshes range from 200-400 grid bars per inch and are made of copper, gold or nickel. A perforated foil with micrometer-sized holes is placed on top of this mesh. Lacey foils provide heterogeneously spaced holes while holey foils are perforated with a predefined hole size, space and arrangement. Copper meshes with carbon foils are commonly used, providing Thon rings for microscope alignment ${ }^{35}$. UltrAuFoil grids are gold meshes with gold foil, which increases electric conduction and heat dissipation, thereby improving stability $^{36}$. Upon vitrification, the difference in thermal expansion between the foil and sample introduces stress ${ }^{37}$, which can result in buckling of the ice layer, leading to beam-induced motion in the microscope, the primary source of resolution loss ${ }^{37}$. The grid geometry is often chosen on the basis of the imaging scheme. The degree of ice buckling can be reduced by decreasing hole sizes to hundreds of nanometers ${ }^{37}$. In contrast, hole sizes in the micrometer range can be used to acquire multiple images per hole.

Some particles have a tendency to stick to the substrate and are unseen in the holes of the foil. Several additions and alterations to sample carriers have been presented to circumvent this and can be roughly classified into two types of approaches: methods to adhere particles to a continuous film and methods to drive particles into the holes. The first group of methods includes addition of a continuous film to which the sample can adhere. Often, these films are made of carbon or graphene (oxide), which can notably increase the number of particles per hole ${ }^{38-40}$. The thickness of these films is generally minimized to avoid additional background noise in microscopy images. It is not trivial to maintain single layers of graphene or graphene oxide sheets ${ }^{41}$; however, success using these films was recently shown with the $52-\mathrm{kDa}$ streptavidin particle, which was resolved to high resolution ${ }^{42,43}$. Moreover, the thin films can be chemically treated to increase binding affinity. Functionalized films, for example, with Ni-NTA, have been shown to bind histidine-tagged particles to increase the number of particles per hole ${ }^{44}$. Antibody binding has also been explored to capture macromolecules in their native state from a cell culture ${ }^{45}$. In addition to increasing the number of particles per hole, these methods have proven advantageous in shielding macromolecules from the air-water interface ${ }^{46}$. The second type of approach deploys chemical treatments to prevent particles from sticking to the foil and push them into the holes. Treatments such as PEGylation have shown potential in distributing particles within the holes of the grid $^{47}$. As no additional film is required, additional background noise in the micrographs is avoided.

To deposit a liquid solution onto a sample carrier, the carrier must be wettable. Plasma treatment is often used in cleaning grids to increase their temporary wettability. In plasma generation, different states of the gas molecules are generated, which are referred to as species. The ratio of the species formed depends on the chamber configuration and operating power, as well as the pressure and flow. The plasma that is generated consists of molecules, ions, 
electrons and radicals ${ }^{48}$. Radicals have an unpaired valence electron, which makes them extremely reactive. Ions, electrons and charged radicals are accelerated toward the electrodes, while molecules and uncharged radicals move in the direction of the gas flow. Therefore, the position of the plasma with respect to the grid affects the amount and type of species that end up at the grid surface. The species etch or bind to the foil, which modifies the surface characteristics of the grid. When ambient air is used in plasma treatment, oxygen and water (depending on the humidity) are the main reactive species, giving the grid an overall negative charge. To increase the reproducibility of the plasma treatment, a controlled mixture of gases can be used $^{49}$. Hydrogen plasma has been used to make plasma treatment gentler. Other compounds, such as amylamine, are used to temporarily give a positive charge to the grid surface ${ }^{50}$.

\section{Deposition}

The ability to deposit a suitable monolayer of macromolecules onto an EM grid still presents an enormous challenge. While success has frequently been achieved for the deposition of well-behaved samples, many iterations are often needed for delicate specimens. To attain fruitful depositions, understanding the influencing factors is key. These factors include shear forces in thin film formations, evaporation, the final layer thickness and molecular dynamics at the various interfaces.

The holes in the foil of the grid have to be filled with a thin layer of liquid. A suitable layer for microscopy is remarkably thin compared to the diameter of the hole itself, for example, a $50-\mathrm{nm}$ layer across a hole of $2 \mu \mathrm{m}$ in diameter ${ }^{51,52}$. Thus, it is important to understand how a thin layer can be formed on an EM grid. Two approaches can be used to fill the holes. In the first, the holes must be completely saturated by a large droplet of liquid to be filled. After saturating the holes through application of a thick liquid layer, wicking is a necessary step to obtain an appropriate layer thickness. In the second approach, liquid is dragged over the perforated foil and the movement fills the holes. With this technique, a thin layer can be formed without the need for additional wicking. During generation of all thin films, the sample encounters shear forces, which originate from a difference in velocity within the liquid. These forces increase in size as the length scale and/or time scale become smaller. It has been hypothesized that shear forces are able to damage fragile proteins $^{53,54}$.

After a thin layer has been deposited on a grid, it is especially susceptible to evaporation because of the high surface-to-volume ratio. Evaporation alters the temperature as well as the $\mathrm{pH}$, salt and particle concentration, carrying the risk of conformational changes to the macromolecule ${ }^{49}$. The amount of evaporation is dependent on the deposition environment and the sample temperature, as well as the duration of exposure. Increasing environmental humidity and/or working at a low temperature, such as $4^{\circ} \mathrm{C}$, can be used to avoid evaporation ${ }^{55}$ and can be done in combination with temperature control of the sample to dewpoint. The dewpoint temperature is the temperature at which the evaporation and condensation of water are in equilibrium. Alternatively, decreasing the elapsed time between deposition and vitrification reduces sample evaporation from the grid.

Precise control over layer thickness at the nanometer scale is key, as thinner ice decreases background noise and may lead to higher-resolution reconstructions ${ }^{56}$. The dimensions of the particle determine a minimum layer thickness. Thinner water layers exclude or denature the particles of interest, while thicker water layers may induce particle overlap. Furthermore, the components in solution influence deposition and wicking behavior. There can be variations in viscosity and density between solutions, and detergents can substantially decrease surface tension ${ }^{56}$. Thus, each combination of desired thickness and fluid properties requires tailored deposition settings.
The dimensions of a thin layer (10-100 $\mathrm{nm}$ in thickness) imply that macromolecules have many more interactions with interfaces than in a bulk solution. Understanding interface reactions is critical to control some of the variability in sample deposition. The airwater interface is a particularly hostile environment for particles. When the hydrophobic region of a macromolecule encounters the air-water interface, this can lead to partial or complete unfolding ${ }^{57}$. The number of particles that denature depends on the total number of interactions as well as their probability of leading to denaturation. The number of interactions has been estimated on the basis of molecular dynamics, using the layer thickness and molecule diameter: for instance, a molecule of $100 \mathrm{kDa}$ in a 100 -nm layer can have 1,000 collisions with interfaces in 1 second, which indicates that multiple interactions will always take place ${ }^{57,58}$. Minimizing the time between deposition and vitrification to tens of milliseconds has been shown to be helpful in preserving protein integrity ${ }^{32}$.

Different methodologies have been introduced to prepare adequate films for single-particle analysis (Table 1). The methods can be classified into three groups: blotting, droplet-based and scribing methods.

Blotting. Blotting is currently the standard and most used technique to create a thin film. It has a long histor ${ }^{59-61}$ and was the only commercial option until recently, with devices such as the Vitrobot (Thermo Fisher Scientific), EM GP (Leica) and Cryoplunge 3 (Gatan). Several protocols for sample preparation involving blotting are available ${ }^{62,63}$ and provide flexibility to the user ${ }^{64}$. In this technique, a droplet of $3-5 \mu \mathrm{l}$ is pipetted onto a hydrophilized grid that is held by sharp tweezers, and a millimeter(s)-thick sample layer fully fills the holes. Filter paper is then used to wick away the excess fluid on the grid to leave a thin sample layer. Upon retraction of the filter paper, the aqueous bridges between the grid and filter paper break. Blotting is typically performed for several seconds in an environment with elevated humidity (Fig. 2a). During this exposure, evaporation as well as the air-water interface may influence sample behavior.

Layer thickness in blotting is controlled using blot time, the properties of the filter papers and blot force. Blot time corresponds to the number of seconds over which the liquid is drawn into the filter paper. The properties of the filter papers determine the capillary suction, which together with the contact area dictates the rate at which the sample is taken up by the papers. As the length scale of the filter paper pores is orders of magnitude larger than the desired layer thickness, this is considered to be one factor that could lead to irreproducibility in layer deposition ${ }^{65}$. In a recent study, there were only a few unpredictable initial contact points between the sample and the filter paper rather than a homogeneous interaction of the blotting paper with sample. The distance between the fibers of the filter paper and the grid is typically several micrometers, leaving space for air to enter. Entrapment of these 'air fingers' is thought to be a locally violent event where shear stresses might reach the order of kilopascals ${ }^{65}$. Finally, tweezers and the location of the grid within the tweezers add variability to the results.

Because blotting instruments can be made on a low budget, modifications have been introduced and are being explored to provide flexible instruments. Blotting devices can provide one-sided/ back-sided blotting for delicate or filamentous structures ${ }^{66,67}$. Applying sample and blotting multiple times to concentrate particles on the grid has also been reported ${ }^{68}$. In combination with binding agents, multiple sample applications can be used to bind samples and wash away residual liquid, creating a purification step on the grid $^{45}$. Time-resolved studies have also been tested through flashlight additions for cage compounds. Light sources placed in the trajectory between the tweezers and the cryogen can be used to capture different conformations in the activation of photolabile compounds in a preset time frame $\mathrm{e}^{69,70}$. 
Table 1 | Deposition techniques

\begin{tabular}{|c|c|c|c|c|c|c|c|c|c|}
\hline $\begin{array}{l}\text { Deposition } \\
\text { techniques }\end{array}$ & Devices & Methodology & $\begin{array}{l}\text { Sample carrier } \\
\text { compatibility }\end{array}$ & $\begin{array}{l}\text { Stock } \\
\text { volume }\end{array}$ & $\begin{array}{l}\text { Volume } \\
\text { per grid }\end{array}$ & $\begin{array}{l}\text { Dewpoint } \\
\text { control }\end{array}$ & $\begin{array}{l}\text { Layer } \\
\text { inspection }\end{array}$ & $\begin{array}{l}\text { Time from } \\
\text { deposition to } \\
\text { vitrification }\end{array}$ & $\begin{array}{l}\text { Grid } \\
\text { coverage }\end{array}$ \\
\hline \multicolumn{10}{|l|}{ Blotting } \\
\hline Pipetting & $\begin{array}{l}\text { Manual plunger }{ }^{15,16} \text {, } \\
\text { Vitrobot, EM GP, } \\
\text { Cryoplunge } 3\end{array}$ & $\begin{array}{l}\text { Wicking of liquid through filter } \\
\operatorname{paper(s)}\end{array}$ & All & $3-5 \mu l$ & $3-5 \mu l$ & No & No & $2-15 s$ & $100 \%$ \\
\hline \multicolumn{10}{|l|}{ Droplet based } \\
\hline \multirow[t]{3}{*}{ Ultrasonic spray } & Back-It-Up ${ }^{83}$ & $\begin{array}{l}\text { High-frequency droplet } \\
\text { generation with through-grid } \\
\text { wicking }\end{array}$ & All & $0.5-1 \mu l$ & $200 \mathrm{nl}-1 \mu \mathrm{l}$ & No & No & $\pm 130 \mathrm{~ms}$ & $25-35 \%$ \\
\hline & Shake-it-off ${ }^{77}$ & $\begin{array}{l}\text { High-frequency droplet } \\
\text { generation with self-wicking } \\
\text { grids }\end{array}$ & $\begin{array}{l}\text { Self-wicking } \\
\text { nanowire grids }\end{array}$ & $0.5-1 \mu l$ & $50 \mathrm{nl}$ & No & No & $<100 \mathrm{~ms}$ & $5-10 \%$ \\
\hline & De Marco group ${ }^{102}$ & $\begin{array}{l}\text { Surface acoustic waves } \\
\text { through microfluidic device }\end{array}$ & All & $0.05-5 \mu l$ & $1.5-2 \mathrm{nl}$ & No & No & $10-1,000 \mathrm{~ms}$ & $5-10 \%$ \\
\hline \multirow[t]{2}{*}{$\begin{array}{l}\text { Gas pressurized } \\
\text { spray }\end{array}$} & $\begin{array}{l}\text { TED (Muench } \\
\text { group) })^{74}\end{array}$ & $\begin{array}{l}\text { Gas sheath around nozzle } \\
\text { using optional high voltage to } \\
\text { steer droplets }\end{array}$ & All & $33 \mu l$ & $4 \mu l$ & No & No & $2-200 \mathrm{~ms}$ & $1-5 \%$ \\
\hline & Frank group ${ }^{72}$ & Gas sheath around nozzle & All & $>30 \mu \mathrm{l}$ & $9 \mu l$ & No & No & $10-1,000 \mathrm{~ms}$ & $5-10 \%$ \\
\hline $\begin{array}{l}\text { Electrostatic } \\
\text { spray }\end{array}$ & Trinick group ${ }^{80}$ & $\begin{array}{l}\text { High potential difference } \\
\text { between nozzle and grid }\end{array}$ & All & $5-10 \mu \mathrm{l}$ & $1-2 \mu l$ & No & No & $>1 \mathrm{~s}$ & $5-10 \%$ \\
\hline Inkjet & $\begin{array}{l}\text { Spotiton }^{81} \\
\text { chameleon }^{85}\end{array}$ & $\begin{array}{l}\text { Droplets formed by a } \\
\text { piezoelectric dispenser } \\
\text { deposited onto self-wicking } \\
\text { grids }\end{array}$ & $\begin{array}{l}\text { Self-wicking } \\
\text { nanowire grids }\end{array}$ & $3-5 \mu l$ & $2-16 \mathrm{nl}$ & No & Yes & $50-2,500 \mathrm{~ms}$ & $10-15 \%$ \\
\hline \multicolumn{10}{|l|}{ Scribing based } \\
\hline Pin printing & VitroJet ${ }^{89}$ & $\begin{array}{l}\text { Dip pen deposition while } \\
\text { maintaining dewpoint }\end{array}$ & All & $0.5 \mu l$ & $1 \mathrm{nl}$ & Yes & Yes & $1-5 s$ & $15-25 \%$ \\
\hline Capillary writing & Cryowriter ${ }^{92}$ & $\begin{array}{l}\text { Capillary deposition with } \\
\text { controlled evaporation or } \\
\text { reaspiration }\end{array}$ & All & $15-25 \mathrm{nl}$ & $0.1 \mathrm{nl}$ & Yes & Yes & $1-3 s$ & $10-20 \%$ \\
\hline
\end{tabular}

Droplet-based methods. While deposition through blotting involves pipetting millimeter-sized droplets, more agile techniques for formation of micrometer-sized droplets have been used in parallel for almost as long ${ }^{71}$. The main motive behind droplet-based techniques has been to capture fast biological processes. Multiple small droplets are generated, which travel and land on a grid. Deposition may take place on a grid that is in trajectory toward the cryogen, resulting in a fast process. Because of the limited processing time, evaporation of the deposited layer is reduced (Fig. 2b).

Generally, 2-40 $\mu \mathrm{l}$ of liquid for deposition is required, owing to the dead volume of the device and the number of droplets that are generated $^{72-74}$. In droplet formation, large shear forces can be present. The higher the velocity and the smaller the droplet, the higher the shear force required for droplet formation ${ }^{75,76}$. Droplet formation can occur through various methods:

1. Ultrasonic spray. An ultrasonic spray can be generated through vibration of an element at very high frequency. Frequencies of over $100 \mathrm{kHz}$ can be used to break up the fluid and generate a spray of droplets of $1-10 \mu \mathrm{m}$ in size $\mathrm{s}^{73,77}$.

2. Gas pressure. The sample solution originates from a nozzle and is surrounded by a high-speed gas sheath. When activated, shear force between the sample and the gas creates a spray of droplets $^{72,78}$. The distribution of droplet diameters can be influenced by changing the gas pressure, resulting in a varying velocity difference between the gas and liquid ${ }^{78}$. To create a more controlled spray, additional charging of the nozzle is possible. By charging the nozzle and sample, the droplet size is more controlled and dispersed, preventing coalescence ${ }^{74}$. A comparison has been made between different sprays with respect to droplet size and thinning on an EM grid ${ }^{79}$.

3. Electrostatic spray. An electrostatic spray can be generated by applying a high potential $(3-10 \mathrm{kV})$ to the sample and grounding the grid. The repulsive forces between the nozzle and sample generate a spray of droplets. This technique was used to generate droplets of $0.25-0.5 \mu \mathrm{m}$ in size that were deposited in combination with blotting ${ }^{80}$.

4. Inkjet. Inkjet printing has been introduced to generate droplets on demand. A pulse train in a piezoelectric dispenser creates a series of single droplets of well-defined diameter $(25 \mu \mathrm{m})$ and direction $^{81}$.

The greatest hurdle in droplet-based methods is spreading of the liquid onto the grid $^{79}$. As the droplet diameter is typically several micrometers, the holes are filled by a relatively thick layer. Upon contact, the deposited droplets spread over the foil and form spherical caps. The peripheries of these caps are sufficiently thin for imaging. The impact of the droplet as well as the hydrophilicity of the substrate determines the shape of the cap and thereby the useful area for imaging ${ }^{72,74,78}$. This approach provides sufficient imaging area for some applications, but imaging area is often increased by additional thinning of the liquid through wicking. Self-wicking grids provide a means to thin the sample at the scale of grid squares. Such grids enhance wicking through nanowires on the grid bars that create a sponge-like effect ${ }^{82}$. Nanowires are made by treating copper-rhodium EM grids with a basic solution of ammonium persulfate, leading to the growth of $\mathrm{Cu}(\mathrm{OH})_{2}$ nanowires ${ }^{82}$. Self-wicking grids are commercially available, while in-house 


\section{NATURE METHODS}

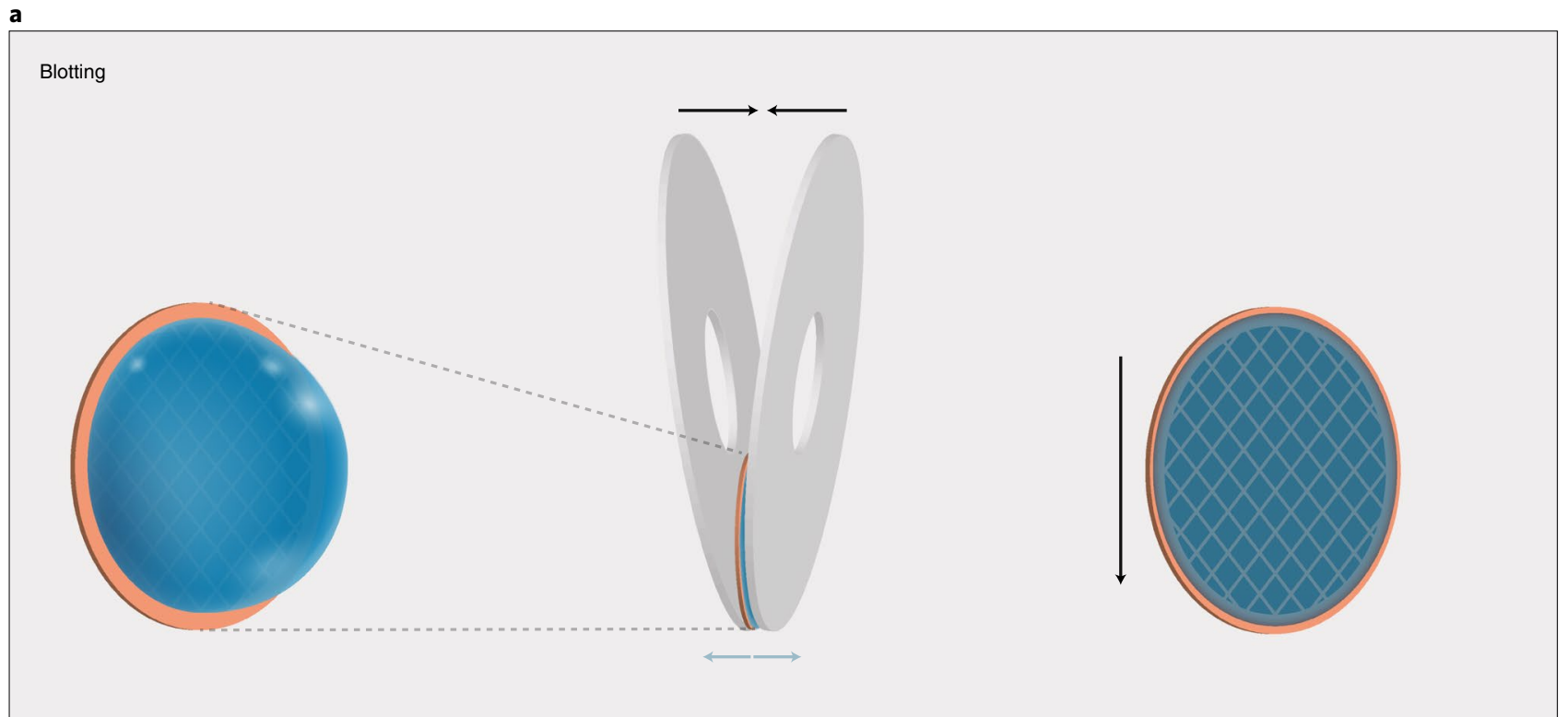

b

Droplet based

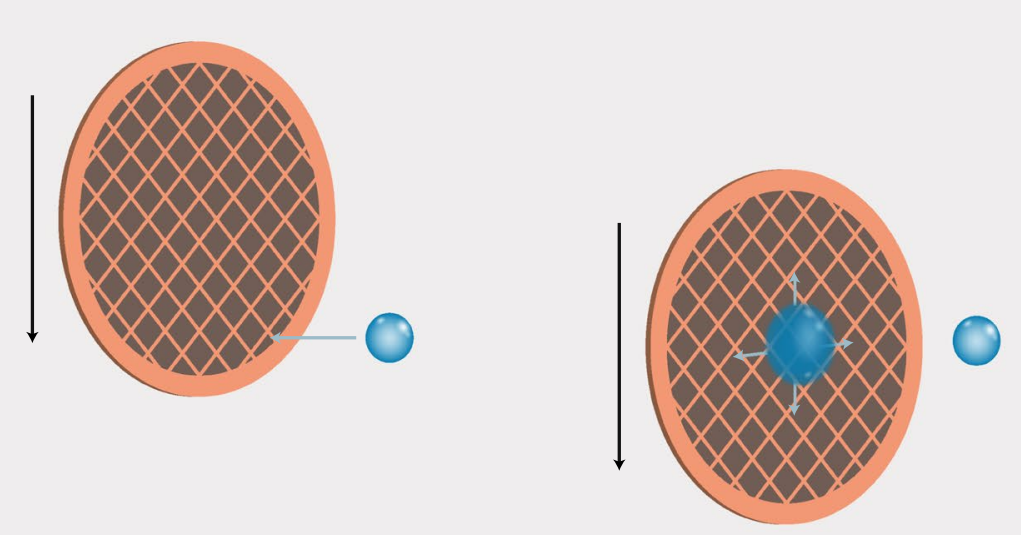


fabrication requires a degree of expertise. The total amount of thinning can be influenced by the volume applied and the capillary suction of the wires as well as the wicking time. Through these measures, thickness can be tuned using wicking time in the millisecond range, where longer exposures result in thinner layers. More recently, a hybrid methodology of droplet-based deposition in combination with back-sided blotting has been demonstrated ${ }^{83}$. In this approach, the sample is deposited using ultrasonic spray and wicked through a grid into a glass fiber filter rather than with nanowires.

The fastest device to date has a time interval between deposition and vitrification of as few as 6 milliseconds ${ }^{84}$. Such fast processes will substantially decrease the number of interactions with the airwater interface $e^{28,51,85}$. This will prove beneficial for many proteins, although instances have been reported where minimizing time negatively impacted the sample ${ }^{84}$. A short time between deposition and vitrification additionally offers vast potential for capturing molecular dynamics. Mixing samples in transit to vitrification may pave the way for analysis of different conformational states in molecular reactions ${ }^{86,87}$.

Scribing. In so-called 'scribing' methods, an element is moved over the grid to deposit a thin layer. The scribing element does not touch the grid but is maintained at a distance of tens of micrometers, defined as the stand-off distance. The sample forms a liquid bridge between the element and the grid. By hovering the element over the grid, the holes are filled (Fig. 2c). The exerted shear forces depend on the scribing velocity and stand-off distance, comparable to the shear between two parallel plates, and have been estimated to be less than 1 pascal ${ }^{88}$. We describe two different scribing-based methods in more detail.

1. Pin printing. In pin printing, a solid metal pin deposits sample using a dip pen method. The solid pin is dipped into a stock solution to collect a subnanoliter droplet on the tip of the pin. The pin is then moved to the grid to form a liquid bridge and deposits a thin layer while moving over the grid surface. During processing, the grid is maintained at the dewpoint temperature to minimize evaporation and condensation of water ${ }^{89}$.

2. Capillary writing. In capillary writing, a sample solution is drawn into a capillary and forced out by gentle pressure to deliver the sample at the grid surface in a scribing motion ${ }^{90}$. Approximately $3-20 \mathrm{nl}$ of sample is deposited onto the grid as a layer, which has to be thinned to obtain a usable layer thickness. Thinning is achieved by adjusting the grid temperature to control evaporation or by reabsorption of excess fluid through the capillary device.

Layer thickness is controlled by the volume introduced to the grid, as well as by the scribing velocity and distance between the element and the grid. The liquid bridge between the scribing element and the grid follows the scribing movement, leaving a trace of sample behind ${ }^{89}$. The width of this trace is related to the diameter of the scribing element, where a larger element allows more grid surface coverage per time unit. The Achilles' heel of scribing is the deposition time, which is one to several seconds. This leads to abundant interactions of the sample with interfaces and requires preservation of the layer during processing ${ }^{89,90}$. By manipulating the grid temperature with respect to the chamber temperature and humidity, evaporation and condensation can be modulated to extend the lifetime of a layer ${ }^{89,90}$. Controlled evaporation can also be used to thin and concentrate the sample ${ }^{90}$.

The scribing motion can be used to deposit sample in a specific pattern and place on the grid. This creates the possibility for deposition of multiple samples where samples may interact. In addition, the scribing technique has been combined with miniaturized protein purification within the scribing element. This approach drastically decreases the amount of cell lysate and purification time required, directly linking proteomics with grid preparation for cryo-EM ${ }^{91}$.

\section{Vitrification}

Following sample deposition on a carrier, the sample must be vitrified and remain in a glass-like state to preserve its interior features, thereby paving the way for reconstructions at high resolution ${ }^{92}$. Rapid cooling is essential to produce vitreous ice and prevent ice crystal formation. The required cooling rates to vitrify water have been estimated to range from $10^{5}$ up to $10^{8} \mathrm{~K}$ per second, implying that there is no clear consensus on the critical cooling rate ${ }^{93-95}$. Addition of cryoprotectants to the sample can decrease the critical cooling rate. The rate that can be achieved is dependent on the type of cryogen, its temperature and the sample. The thermal and physical properties of the cryogen dictate its cooling efficiency. Cryogens tested for cryo-EM include ethane, ethane-propane mixtures and freon as well as slush nitrogen. A comparison has been done showing that liquid ethane has the best relative cooling efficiency in single-particle studies $^{96}$. Ethane-propane mixtures have been used as they do not solidify when at the temperature of liquid nitrogen and have been shown to have a larger cooling capacity for thicker samples ${ }^{97}$.

Currently, plunging a grid into a bath of liquid cryogen, known as plunge freezing, is widely used to vitrify samples of $1 \mu \mathrm{m}$ or thinner (Fig. 3a). This technique has been the primary method of vitrification since its proof of concept with water ${ }^{15}$. When a grid is plunged into a bath, the grid circumference makes the first contact with the cryogen. Heat conduction through the grid bars and foil leads to vitrification of the sample ${ }^{98}$. After vitrification, grids have to be handled below $-137^{\circ} \mathrm{C}$ to prevent devitrification of the ice layer ${ }^{15,59}$. The grids are then loaded into a side entry holder or are mounted in a cartridge for further batch processing. This includes automated specimen handling at low temperature inside the microscope. The mounting procedure can be considered a hassle for many novice users as clipping must be performed at liquid nitrogen temperatures ${ }^{62,63}$. Any handling of grids during or after vitrification runs the risk of contaminating the precious frozen samples with ice crystals.

Recently, jet vitrification has been described to vitrify samples deposited on preclipped autogrids (Fig. 3b). Autogrids are an ensemble of the EM grid held within a sturdy copper ring by means of a clip. The grids can then be automatically handled in high-end microscopes containing autoloaders. Jet freezing was originally designed for tissue fixation and subsequent freeze substitution ${ }^{99}$. In jet freezing for cryo-EM, two cryogen jets are directed toward the center of the grid, aiming at the deposited sample. With continued cooling by the jets, the cryogen spreads out, thereby completely cooling the grid together with the bulky autogrid ring. Measurements have shown that higher cooling rates are achieved by jet freezing than with plunge freezing ${ }^{89}$.

\section{Outlook}

Cryo-EM is evolving to become a go-to structural biology technique, provided that sample preparation keeps up with demand. Structural biologists with different backgrounds have already converted to the field as it offers promise for new structures at high resolution. Understanding the role of different macromolecular structures will require a push for workflow maturation. Cryo-EM is moving at a fast pace, where results can quickly be obtained once the right settings are determined ${ }^{24}$. Faster cameras have been developed to collect more images over a shorter period of time. Screening microscopes are in high demand so more grids can be prepared and checked. Software is being developed to increase throughput in different steps of data processing ${ }^{24}$. Collecting excess data and only later filtering out usable particles is the current strategy ${ }^{100}$. However, this strategy does not address the problems of robust and reliable specimen preparation. The speed of developments has put 
a

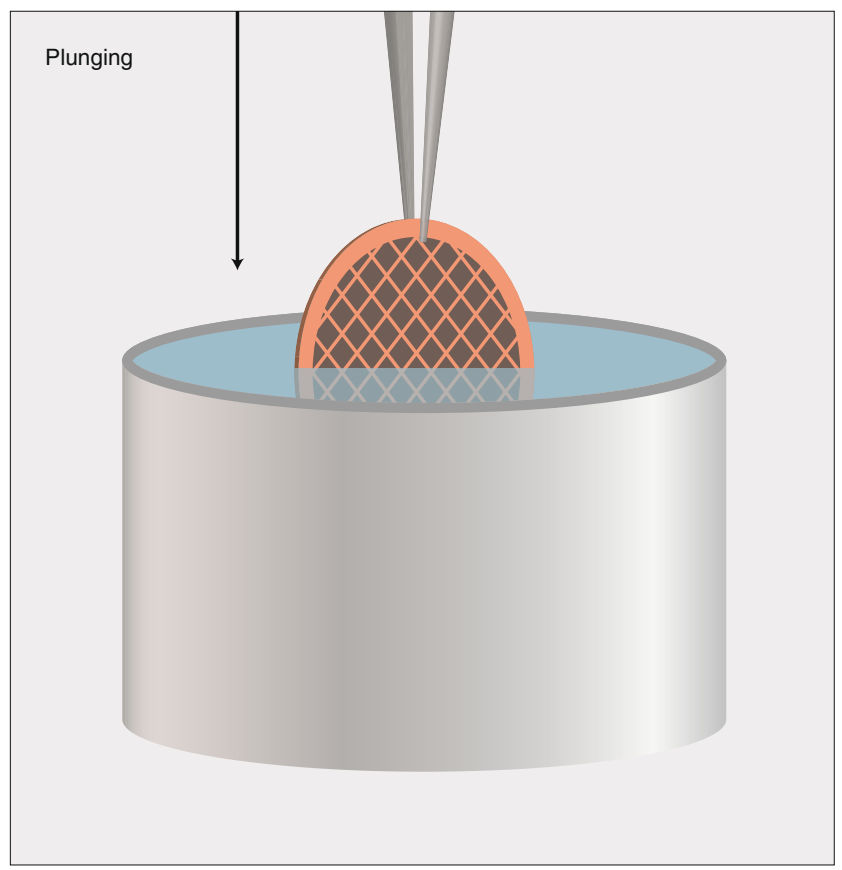

b

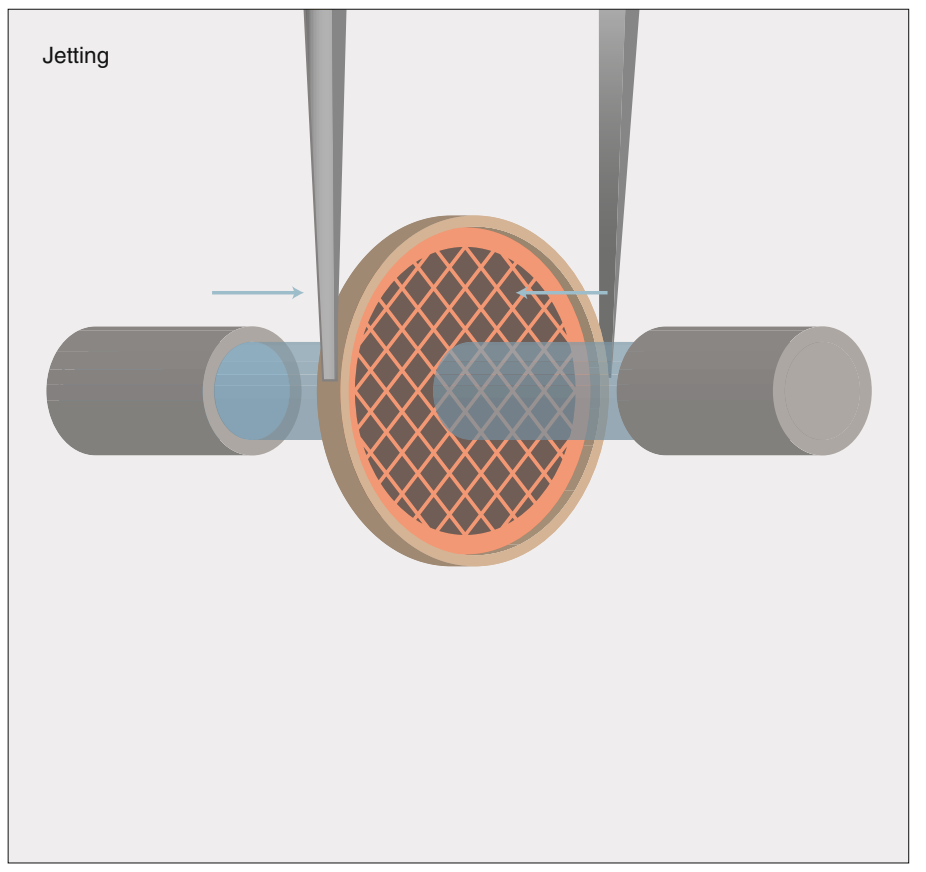

Fig. 3 | Two ways of vitrifying EM grids. a, Plunge freezing involves immersing a grid in a pool of cryogen (black arrow) where the edge of the grid first makes contact followed by the rest of the grid. $\mathbf{b}$, Jet vitrification entails a preclipped autogrid that is held before two jets of cryogen targeting the center of the grid (blue arrows).

the spotlight on the meticulous and time-consuming number of iterations needed to adjust sample properties. Many rounds of optimization are often needed before a suitable molecule is obtained for data collection ${ }^{14}$.

Sample preparation must increase in yield and reach a high standard to keep pace with community demand. Easier, soluble proteins have already been resolved or are being resolved now, leaving many complex, heterogeneous samples to be the candidates of future studies $^{19,101}$. At this stage, grid preparation is approached from different angles to learn which of the numerous options is most suitable for a particular sample. Several divergent methods are bundled in devices entering the market ${ }^{85,89}$ or have been introduced experimentally in labs $^{72,77,80,83,84,87,90,102}$. Miniaturization has pushed for greater efficiency in the overall workflow. New technologies focusing on control and throughput will undoubtedly prove valuable to users trying to optimize their microscope usage. Speeding up the transition from deposition to vitrification has already been shown to have value in the battle against sample denaturation. At the same time, there is a pull toward more flexible, creative, low-cost techniques without the ease of use of commercial frameworks ${ }^{77}$. Because it is still difficult to pinpoint the exact issues samples encounter on an EM grid, more platforms will surely provide more answers.

The field has the momentum to learn from the upcoming developments and alleviate the bottlenecks in grid preparation. Because cryo-EM infrastructure is already available, a big leap can be taken once sample preparation yield increases and microscopes are used more efficiently. In this phase, streamlining and throughput will have a crucial role in the process, as they do in X-ray crystallography ${ }^{103}$. The foundations of cryo-EM data management are in the works and will be imperative as more scientists contribute to the pursuit of structures ${ }^{104}$.

While boundaries are continuously being pushed, there are still limitations in the technique. Size does matter in cryo-EM. The majority of small structures that are often targets in drug discovery are not yet solvable by cryo-EM and are still being resolved through crystallography ${ }^{105}$. At the moment, structures as small as the $52-\mathrm{kDa}$ streptavidin and the $40-\mathrm{kDa}$ SAM-IV riboswitch RNA ${ }^{106}$ are the practical limits ${ }^{107}$. With the expansion of the field and new developments, it is foreseeable that closely related techniques will evolve as well. Time-resolved microscopy will likely be used more frequently to observe different macromolecular states ${ }^{87,102,108-110}$. In addition, the behavior of macromolecules in their native environment is of tremendous interest for many. Cellular cryo-electron tomography, known for its intricate workflow, is already benefiting from the groundwork laid for single-particle analysis ${ }^{111}$. Given the rate of advancements in the field, we are optimistic that sample preparation will accelerate in line with demand. Demystification of the invisible hands in sample preparation will propel cryo-EM as a standard technique in structural biology.

Received: 11 September 2020; Accepted: 30 March 2021; Published online: 7 May 2021

\section{References}

1. Fitzpatrick, A. W. P. et al. Cryo-EM structures of tau filaments from Alzheimer's disease. Nature 547, 185-190 (2017).

2. Gremer, L. et al. Fibril structure of amyloid- $\beta_{1-42}$ by cryo-electron microscopy. Science 358, 116-119 (2017).

3. Wrapp, D. et al. Cryo-EM structure of the $2019-\mathrm{nCoV}$ spike in the prefusion conformation. Science 367, 1260-1263 (2020).

4. Shang, J. et al. Structure of mouse coronavirus spike protein complexed with receptor reveals mechanism for viral entry. PLoS Pathog. 16, e1008392 (2020).

5. Walls, A. C. et al. Structure, function, and antigenicity of the SARS-CoV-2 spike glycoprotein. Cell 181, 281-292 (2020).

6. Renaud, J. P. et al. Cryo-EM in drug discovery: achievements, limitations and prospects. Nat. Rev. Drug Discov. 17, 471-492 (2018).

7. Kühlbrandt, W. The resolution revolution. Science 343, 1443-1444 (2014)

8. Faruqi, A. R. \& Henderson, R. Electronic detectors for electron microscopy. Curr. Opin. Struct. Biol. 17, 549-555 (2007).

9. Brilot, A. F. et al. Beam-induced motion of vitrified specimen on holey carbon film. J. Struct. Biol. 177, 630-637 (2012). 
10. Baldwin, P. R. et al. Big data in cryoEM: automated collection, processing and accessibility of EM data. Curr. Opin. Microbiol. 43, 1-8 (2018).

11. Maruthi, K., Kopylov, M. \& Carragher, B. Automating decision making in the cryo-EM pre-processing pipeline. Structure 28, 727-729 (2020).

12. Nakane, T. et al. Single-particle cryo-EM at atomic resolution. Nature $\mathbf{5 8 7}$ 152-156 (2020).

The authors accelerate data acquisition and improve sample quality through technological advancements. This opens the door to routine high-resolution and high-throughput cryo-EM.

13. Yip, K. M., Fischer, N., Paknia, E., Chari, A. \& Stark, H. Atomic-resolution protein structure determination by cryo-EM. Nature 587, 157-161 (2020). Substantial improvement in microscope hardware lead to visualization of individual atoms in a protein. Cryo-EM density maps of this quality can be applicable for structure-based drug design.

14. Carragher, B. et al. Current outcomes when optimizing 'standard' sample preparation for single-particle cryo-EM. J. Microsc. 276, 39-45 (2019). This overview of sample preparation methods can serve as a guideline for new users or when experiencing difficulties with novel specimens.

15. Dubochet, J. \& McDowall, A. W. Vitrification of pure water for electron microscopy. J. Microsc. 124, RP3-RP4 (1981).

A method for preparing vitrified ice in liquid propane or ethane for direct observation in the electron microscope is shown for the first time.

16. Herzik, M. A., Wu, M. \& Lander, G. C. Achieving better-than-3-A resolution by single-particle cryo-EM at $200 \mathrm{keV}$. Nat. Methods 14, 1075-1078 (2017).

17. Tan, Y. Z. et al. Sub-2 ̊ Ewald curvature corrected structure of an AAV2 capsid variant. Nat. Commun. 9, 3628 (2018).

18. Herzik, M. A., Wu, M. \& Lander, G. C. High-resolution structure determination of sub-100 kDa complexes using conventional cryo-EM. Nat. Commun. 10, 1032 (2019)

19. Serna, M. Hands on methods for high resolution cryo-electron microscopy structures of heterogeneous macromolecular complexes. Front. Mol. Biosci. 6, 33 (2019).

20. Drulyte, I. et al. Approaches to altering particle distributions in cryoelectron microscopy sample preparation. Acta Crystallogr. D Struct. Biol. 74, 560-571 (2018).

21. Glaeser, R. M. How good can cryo-EM become? Nat. Methods 13, 28-32 (2016)

22. Kim, L. Y. et al. Benchmarking cryo-EM single particle analysis workflow. Front. Mol. Biosci. 5, 50 (2018).

23. Lander, G. C. et al. Appion: an integrated, database-driven pipeline to facilitate EM image processing. J. Struct. Biol. 166, 95-102 (2009).

24. Li, Y., Cash, J. N., Tesmer, J. J. G. \& Cianfrocco, M. A. High-throughput cryo-EM enabled by user-free preprocessing routines. Structure $\mathbf{2 8}$, 858-869 (2020).

25. Callaway, E. The revolution will not be crystallized: a new method sweeps through structural biology. Nature 525, 172-174 (2015).

26. Autzen, H. E., Julius, D. \& Cheng, Y. Membrane mimetic systems in CryoEM: keeping membrane proteins in their native environment. Curr. Opin. Struct. Biol. 58, 259-268 (2019).

27. Kumar, A. et al. Mechanisms of activation and desensitization of full-length glycine receptor in lipid nanodiscs. Nat. Commun. 11, 3752 (2020).

28. Chen, J., Noble, A. J., Kang, J. Y. \& Darst, S. A. Eliminating effects of particle adsorption to the air/water interface in single-particle cryo-electron microscopy: bacterial RNA polymerase and CHAPSO. J. Struct. Biol. X 1, 100005 (2019).

29. Chu, F., Thornton, D. T. \& Nguyen, H. T. Chemical cross-linking in the structural analysis of protein assemblies. Methods 144, 53-63 (2018).

30. Stark, H. GraFix: stabilization of fragile macromolecular complexes for single particle cryo-EM. Methods Enzymol. 481, 109-126 (2010).

31. Gewering, T., Januliene, D., Ries, A. B. \& Moeller, A. Know your detergents: a case study on detergent background in negative stain electron microscopy. J. Struct. Biol. 203, 242-246 (2018).

32. Noble, A. J. et al. Reducing effects of particle adsorption to the air-water interface in cryo-EM. Nat. Methods 15, 793-795 (2018).

The authors show that damaging effects of particle adsorption to airwater interfaces can be reduced by minimizing the time between deposition and freezing.

33. Bokori-Brown, M. et al. Cryo-EM structure of lysenin pore elucidates membrane insertion by an aerolysin family protein. Nat. Commun. 7, 11293 (2016)

34. Karuppasamy, M., Karimi Nejadasl, F., Vulovic, M., Koster, A. J. \& Ravelli, R. B. G. Radiation damage in single-particle cryo-electron microscopy: effects of dose and dose rate. J. Synchrotron Radiat. 18, 398-412 (2011).

35. Ermantraut, E., Wohlfart, K. \& Tichelaar, W. Perforated support foils with pre-defined hole size, shape and arrangement. Ultramicroscopy 74, 75-81 (1998).

36. Russo, C. J. \& Passmore, L. A. Ultrastable gold substrates: properties of a support for high-resolution electron cryomicroscopy of biological specimens. J. Struct. Biol. 346, 1377-1380 (2016).
37. Naydenova, K., Jia, P. \& Russo, C. J. Cryo-EM with sub-1 A specimen movement. Science 370, 223-226 (2020).

The authors introduce a new specimen support that minimizes beam-induced motion of the ice layer, reducing information loss

38. Russo, C. J. \& Passmore, L. A. Controlling protein adsorption on graphene for cryo-EM using low-energy hydrogen plasmas. Nat. Methods 11, 649-652 (2014).

39. Rawson, S. et al. Structure of the vacuolar $\mathrm{H}^{+}$-ATPase rotary motor reveals new mechanistic insights. Structure 22, 461-471 (2015).

40. Palovcak, E. et al. A simple and robust procedure for preparing graphene-oxide cryo-EM grids. J. Struct. Biol. 204, 80-84 (2018).

41. Naydenova, K., Peet, M. J. \& Russo, C. J. Multifunctional graphene supports for electron cryomicroscopy. Proc. Natl Acad. Sci. USA 116 , 11718-11724 (2019)

42. Fan, X. et al. Single particle cryo-EM reconstruction of $52 \mathrm{kDa}$ streptavidin at 3.2 Angstrom resolution. Nat. Commun. 10, 2386 (2019).

43. Han, Y. et al. High-yield monolayer graphene grids for near-atomic resolution cryoelectron microscopy. Proc. Natl Acad. Sci. USA 117, 1009-1014 (2020)

44. Llaguno, M. C. et al. Chemically functionalized carbon films for single molecule imaging. J. Struct. Biol. 185, 405-417 (2014).

45. Yu, G., Li, K. \& Jiang, W. Antibody-based affinity cryo-EM grid. Methods 100, 16-24 (2016).

46. D'Imprima, E. et al. Protein denaturation at the air-water interface and how to prevent it. eLife 8, e42747 (2019).

47. Meyerson, J. R. et al. Self-assembled monolayers improve protein distribution on holey carbon cryo-EM supports. Sci. Rep. 4, 7084 (2014).

48. Belkind, A. \& Gershmans, S. Plasma cleaning of surfaces. Vac. Technol. Coat. 2008, 46-57 (2008).

49. Passmore, L. A. \& Russo, C. J. Specimen preparation for high-resolution cryo-EM. Methods Enzymol. 579, 51-86 (2016).

50. Craig, L. et al. Type IV pilus structure by cryo-electron microscopy and crystallography: implications for pilus assembly and functions. Mol. Cell 23, 651-662 (2006).

51. Noble, A. J. et al. Routine single particle cryoEM sample and grid characterization by tomography. eLife 7, e34257 (2018).

52. Rice, W. J. et al. Routine determination of ice thickness for cryo-EM grids. J. Struct. Biol. 204, 38-44 (2018).

53. Glaeser, R. M. Proteins, interfaces, and cryo-EM grids. Curr. Opin. Colloid Interface Sci. 34, 1-8 (2018).

54. Zheng, Y. et al. Cryo-TEM imaging the flow-induced transition from vesicles to threadlike micelles. J. Phys. Chem. B 104, 5263-5271 (2000).

55. Frederik, P. M. \& Hubert, D. H. W. Cryoelectron microscopy of liposomes. Methods Enzymol. 391, 431-448 (2005).

The authors explain that a thin film is vulnerable to osmotic and temperature-induced alterations, which can be avoided by controlling the environmental temperature and humidity.

56. Glaeser, R. M. et al. Factors that influence the formation and stability of thin, cryo-EM specimens. Biophys. J. 110, 749-755 (2016).

57. Taylor, K. A. \& Glaeser, R. M. Retrospective on the early development of cryoelectron microscopy of macromolecules and a prospective on opportunities for the future. J. Struct. Biol. 163, 214-223 (2008).

58. Naydenova, K. \& Russo, C. J. Measuring the effects of particle orientation to improve the efficiency of electron cryomicroscopy. Nat. Commun. 8 , 629 (2017)

59. Dubochet, J., Lepault, J., Freeman, R., Berriman, J. A. \& Homo, J. -C. Electron microscopy of frozen water and aqueous solutions. J. Microsc. 128, 219-237 (1982).

The authors demonstrate the structure of various samples and the influence of the electron beam on them.

60. Bellare, J. R., Davis, H. T., Scriven, L. E. \& Talmon, Y. Controlled environment vitrification system: an improved sample preparation technique. J. Electron Microsc. Tech. 10, 87-111 (1988).

61. Frederik, P. M., Stuart, M. C. A. \& Verkleij, A. J. Intermediary structures during membrane fusion as observed by cryo-electron microscopy. Biochim. Biophys. Acta 979, 275-278 (1989).

62. Grassucci, R. A., Taylor, D. J. \& Frank, J. Preparation of macromolecular complexes for cryo-electron microscopy. Nat. Protoc. 2, 3239-3246 (2007).

63. Thompson, R. F., Walker, M., Siebert, C. A., Muench, S. P. \& Ranson, N. A An introduction to sample preparation and imaging by cryo-electron microscopy for structural biology. Methods 100, 3-15 (2016).

64. Frederik, P. M., De Haas, F. \& Storms, M. M. H. Controlled vitrification. in Handbook of Cryo-Preparation Methods for Electron Microscopy (eds. Cavalier, A., Spehner, D. \& Humbel, B. M.) 71-102 (CRC, 2009).

65. Armstrong, M. et al. Micro-scale fluid behavior during cryo-EM sample blotting. Biophys. J. 118, 708-719 (2019).

66. Amos, L. A. \& Hirose, K. Studing the structure of microtubules by electron microscopy. Microtubule Protoc. 137, 65-91 (2007) 
67. Jakobi, A. J. et al. Structural basis of p62/SQSTM1 helical filaments and their role in cellular cargo uptake. Nat. Commun. 11, 440 (2020).

68. Snijder, J. et al. Vitrification after multiple rounds of sample application and blotting improves particle density on cryo-electron microscopy grids. J. Struct. Biol. 198, 38-43 (2017).

69. Ménétret, J. F., Hofmann, W., Schröder, R. R., Rapp, G. \& Goody, R. S Time-resolved cryo-electron microscopic study of the dissociation of actomyosin induced by photolysis of photolabile nucleotides. J. Mol. Biol. 219, 139-144 (1991).

70. Subramaniam, S., Gerstein, M., Oesterhelt, D. \& Henderson, R. Electron diffraction analysis of structural changes in the photocycle of bacteriorhodopsin. EMBO J. 12, 1-8 (1993).

71. Berriman, J. \& Unwin, N. Analysis of transient structures by cryo-microscopy combined with rapid mixing of spray droplets. Ultramicroscopy 56, 241-252 (1994).

72. Kaledhonkar, S., Fu, Z., White, H. \& Frank, J. Time-resolved cryo-electron microscopy using a microfluidic chip. Methods Mol. Biol. 1764, 59-71 (2018).

73. Ashtiani, D. et al. Delivery of femtolitre droplets using surface acoustic wave based atomisation for cryo-EM grid preparation. J. Struct. Biol. 203, 94-101 (2018).

74. Kontziampasis, D., Klebl, D. P., Iadanza, M. G., Scarff, C. A. \& White, H. D. A cryo-EM grid preparation device for time-resolved structural studies. IUCrJ 6, 1024-1031 (2019).

75. Avvaru, B., Patil, M. N., Gogate, P. R. \& Pandit, A. B. Ultrasonic atomization: effect of liquid phase properties. Ultrasonics 44, 146-158 (2006).

76. Driessen, T. Drop Formation from Axi-Symmetric Fluid Jets. PhD thesis, Universiteit Twente (2013).

77. Rubinstein, J. L. et al. Shake-it-off: a simple ultrasonic cryo-EM specimenpreparation device. Acta Crystallogr. D Struct. Biol. 75, 1063-1070 (2019).

78. Feng, X. et al. A fast and effective microfluidic spraying-plunging method for high-resolution single-particle cryo-EM. Structure 25, 663-670 (2017).

79. Klebl, D. P. et al. Sample deposition onto cryo-EM grids: from sprays to jets and back. Acta Crystallogr. D Struct. Biol. 76, 340-349 (2020).

80. White, H. D., Thirumurugan, K., Walker, M. L. \& Trinick, J. A second generation apparatus for time-resolved electron cryo-microscopy using stepper motors and electrospray. J. Struct. Biol. 144, 246-252 (2003).

81. Jain, T., Sheehan, P., Crum, J., Carragher, B. \& Potter, C. S. Spotiton: a prototype for an integrated inkjet dispense and vitrification system for cryo-TEM. J. Struct. Biol. 179, 68-75 (2012).

82. Wei, H. et al. Self-blotting nanowire grids for cryo-EM sample preparation. Microsc. Microanal. 23, 848-849 (2017).

83. Tan, Y. Z. \& Rubinstein, J. L. Through-grid wicking enables high-speed cryoEM specimen preparation. Acta Crystallogr. D Struct. Biol. 76, 1092-1103 (2020).

84. Klebl, D. P. et al. Need for speed: examining protein behaviour during cryoEM grid preparation at different timescales. Structure 28, 1238-1248 (2020). The authors present a comparison of different deposition techniques in relation to the time between deposition and vitrification. The effect on particle distribution and concentration in the ice layer is examined

85. Darrow, M. C., Moore, J. P., Walker, R. J., Doering, K. \& King, R. S. Chameleon: next generation sample preparation for cryoEM based on Spotiton. Microsc. Microanal. 25, $994-995$ (2019).

86. Chen, B. et al. Structural dynamics of ribosome subunit association studied by mixing-spraying time-resolved cryogenic electron microscopy. Structure 23, 1097-1105 (2015).

87. Dandey, V. P. et al. Time-resolved cryo-EM using Spotiton. Nat. Methods 17, 897-900 (2020).

88. Neto, C., Evans, D. R., Bonaccurso, E., Butt, H. J. \& Craig, V. S. J. Boundary slip in Newtonian liquids: a review of experimental studies. Rep. Prog. Phys. 68, 2859 (2005).

89. Ravelli, R. B. G. et al. Cryo-EM structures from sub-nl volumes using pin-printing and jet vitrification. Nat. Commun. 11, 2563 (2020).

90. Arnold, S. A. et al. Blotting-free and lossless cryo-electron microscopy grid preparation from nanoliter-sized protein samples and single-cell extracts. J. Struct. Biol. 197, 220-226 (2017).

91. Schmidli, C. et al. Microfluidic protein isolation and sample preparation for high-resolution cryo-EM. Proc. Natl Acad. Sci. USA 116, 15007-15012 (2019).

92. Frank, J. Three-Dimensional Electron Microscopy of Macromolecular Assemblies: Visualization of Biological Molecules in Their Native State (Oxford University Press, 2006).

93. Fletcher, N. H. Structural aspects of the ice-water system. Rep. Prog. Phys. 34, 913 (1971)
94. Tyree, T. J., Dan, R. \& Thorne, R. E. Density and electron density of aqueous cryoprotectant solutions at cryogenic temperatures for optimized cryoprotection and diffraction contrast. Acta Crystallogr. D Struct. Biol. 74, 471-479 (2018)

95. Vanhecke, D. et al. Cryo-electron tomography: methodology, developments and biological applications. J. Microsc. 242, 221-227 (2011).

96. Cheng, D., Mitchell, D., Shieh, D. \& Braet, F. Practical considerations in the successful preparation of specimens for thin-film cryo-transmission electron microscopy. Curr. Microsc. Contrib. Adv. Sci. Technol. 2, 880-890 (2012).

97. Tivol, W. F., Briegel, A. \& Jensen, G. J. An improved cryogen for plunge freezing. Microsc. Microanal. 14, 375 (2008).

98. Kasas, S., Dumas, G., Dietler, G., Catsicas, S. \& Adrian, M. Vitrification of cryoelectron microscopy specimens revealed by high-speed photographic imaging. J. Microsc. 211, 48-53 (2003).

99. Burstein, N. L., \& Maurice, D. M. Cryofixation of tissue surfaces by a propane jet for electron microscopy. Micron 9, 191-198 (1978).

100. Hartmann, C. Industrial cryo-EM facility setup and management. Acta Crystallogr. D Struct. Biol. 76, 313-325 (2020).

101. Cheng, Y. Single-particle cryo-EM-how did it get here and where will it go. Science 361, 876-880 (2018).

102. Ashtiani, D., De Marco, A. \& Neild, A. Tailoring surface acoustic wave atomisation for cryo-electron microscopy sample preparation. Lab Chip 19 , 1378-1385 (2019)

103. Merk, A. et al. Breaking cryo-EM resolution barriers to facilitate drug discovery. Cell 165, 1698-1707 (2016).

104. Estevez, A. et al. Building cryo-EM at Genentech to enable research and drug discovery. Microsc. Microanal. 25, 1314-1315 (2019).

105. Shi, Y. A glimpse of structural biology through X-ray crystallography. Cell 159, 995-1014 (2014).

106. Zhang, K. et al. Cryo-EM structure of a $40 \mathrm{kDa}$ SAM-IV riboswitch RNA at $3.7 \AA$ resolution. Nat. Commun. 10, 5511 (2019).

107. Lyumkis, D. Challenges and opportunities in cryo-EM single-particle analysis. J. Biol. Chem. 294, 5181-5197 (2019).

108. Fu, Z. et al. The structural basis for release-factor activation during translation termination revealed by time-resolved cryogenic electron microscopy. Nat. Commun. 10, 2579 (2019).

109. Kaledhonkar, S. et al. Late steps in bacterial translation initiation visualized using time-resolved cryo-EM. Nature 570, 400-404 (2019).

110. Dance, A. Molecular motion on ice. Nat. Methods 17, 879-883 (2020).

111. Lučić, V., Rigort, A. \& Baumeister, W. Cryo-electron tomography: the challenge of doing structural biology in situ. J. Cell Biol. 202, 407-419 (2013).

\section{Acknowledgements}

This manuscript is dedicated to Dominique Hubert, who participated in Vitrobot and cryo-EM instrument development and who passed away much too young. We would like to thank S. Muench, J. Frank, H. White, A. de Marco, J. Rubinstein, B. Carragher, M. Darrow and T. Braun for providing detailed input and reviewing the table. We thank R. Ravelli and F. Nijpels for helpful discussions and W. Hagen, P. Frederik, H. Nguyen and A. Gijsbers for critically reading the manuscript. Finally, we thank M. de Backer and J. Dabekaussen for contributing to the figures. This work received funding from the Netherlands Organization for Scientific Research (NWO) in the framework of the Fund New Chemical Innovations, numbers 731.014.109 and 731.016.407, as well as from the province of Limburg, the Netherlands.

\section{Competing interests}

The University of Maastricht has filed patents with R.J.M.H. and P.J.P. as inventors regarding sample preparation for cryo-EM. G.W. and R.J.M.H. are employed by and P.J.P. is a shareholder of CryoSol-World, which holds the licenses for these submitted patents.

\section{Additional information}

Correspondence should be addressed to P.J.P.

Peer review information Nature Methods thanks Mark Herzik Jr and the other, anonymous, reviewer(s) for their contribution to the peer review of this work. Arunima Singh was the primary editor on this article and managed its editorial process and peer review in collaboration with the rest of the editorial team.

Reprints and permissions information is available at www.nature.com/reprints.

Publisher's note Springer Nature remains neutral with regard to jurisdictional claims in published maps and institutional affiliations.

(c) Springer Nature America, Inc. 2021 